SHUGUANG LI

E-mail: Ixlsg@vip.sina.com

Chang'an University

School of Electronic and Control Engineering

Xi'an 710064, PR China
Science in Traffic and Transport

Preliminary Communication

Accepted: May 4, 2011

Approved: Oct. 3, 2012

\title{
A BASED-BOTTLENECK MULTIPLE VEHICLE TYPE DYNAMIC MARGINAL COST MODEL AND ALGORITHM
}

\begin{abstract}
Single vehicle type dynamic marginal cost model is extended to multiple vehicle type dynamic one based on time-dependent multiple vehicle type queue analysis at a bottleneck. A dynamic link model is presented to model interactions between cars and trucks, given the link consists of two distinct segments. The first segment is the running segment on which cars (trucks) run at their free-flow speeds and the second segment is the exit queue segment. A car or a truck is assumed to be a point without length. The classspecific pi parameter is used to transform the effect of truck into passenger car equivalents, so the exit flow of cars and trucks can be calculated according to the exit capacity of a bottleneck. The analytic expression of multiple vehicle type dynamic marginal cost function is deduced under congested and uncongested conditions. Then a heuristic algorithm is presented in solving multiple vehicle type dynamic queues, tolls under system optimum and user equilibrium conditions. The numerical example illustrates the simplicity and applicability of the proposed approach.
\end{abstract}

\section{KEYWORDS}

multiple vehicle types, dynamic marginal cost function, point queue, traffic bottleneck

\section{INTRODUCTION}

The congestion pricing method to reduce traffic congestion has attracted the attention in many countries. Some congestion pricing projects have been implemented in Singapore and more recently, in London. Previous studies have focused on single user class dynamic congestion pricing [1-3]. The implementation of congestion pricing policy faces an important issue in estimating the toll of different user types (cars, trucks, etc.) that differences among multiple user types result in the different externalities in terms of congestion and pollution emissions.

Arnott et al. used a simple static congestion model in which the users with different values of times choose between two parallel routes, and gave the optimal tolls for the integrated and separated usage for different users [4]. Verhoef et al. proposed a dynamic toll function in considering the vehicles with speed difference [5]. Holgun et al. used a micro-simulation method in modelling the interactions of different vehicle types to estimate the valuation of externalities such as congestion, pavement deterioration, and environmental impacts to compute optimal tolls for multi-class traffic [6]. Li et al. presented a multiple vehicle type dynamic marginal travel cost model in considering departure time choices [11].

In this paper, following the work of Li et al. in [11], a new analytical dynamic marginal cost function is given without considering the departure time choices. It is proved that optimal tolls for trucks are higher than those for cars; furthermore, the reasons for traffic congestion reduced by congestion pricing method without departure time choices is analyzed under traffic conditions with multiple vehicle types.

The rest of the paper is organized as follows. Firstly, we introduce a multiple vehicle type dynamic bottleneck model in considering dynamic queues. Secondly, single vehicle type dynamic marginal cost analysis is extended to multiple vehicle type dynamic one based on the time-dependent multiple vehicle type queue analysis at a bottleneck link. Furthermore, an analytical multiple vehicle type dynamic marginal cost function is presented under uncongested and congested conditions. Finally, a simple heuristic algorithm is given in calculating multiple vehicle type dynamic tolls and the example is used to analyze the properties of the model.

\section{MULTIPLE VEHICLE TYPE DYNAMIC BOTTLENECK MODELS}

Let us consider a bottleneck link with a fixed exit capacity s, expressed in passenger car unit per hour: 
$P c u / h$. Let us assume there are two vehicle types, cars and trucks. Li et al. in [7-8] proposed a multimode stochastic dynamic simultaneous route/departure time equilibrium model and proved the multimode deterministic point model where each mode-user meets the First-in-First-out (FIFO) discipline and the speeds of different modes approach consistent during congestion [9-10]. A similar point queue model proposed by Li et al. in [11] is used in the paper.

The link flow propagation on a bottleneck link is shown in Figure 1 (a). Cars and trucks have the typespecific characteristics, such as free flow speed and vehicle size. A bottleneck link consists of two distinct segments. On the first running segment on a bottleneck link, cars (trucks) run according to type-specific speeds. The second segment is the exit queue segment (a car or a truck is assumed to be a point without length). If the type-specific $p_{i}$ parameter is used to transform the effect of truck into passenger car equivalents, then the exit flow of cars and trucks can be calculated according to the exit capacity s (in $P c u / h$ ) of a bottleneck link. Figure 1 (b) gives the change of total cumulative trips (in $\mathrm{Pcu}$ ) with respect to time. The following parts give a link flow model in considering the interaction of cars and trucks on a bottleneck link.

\section{Link Dynamic Function}

The flow rate of car (truck) travellers entering a bottleneck link at moment $t-t_{i}$ is $u_{i}\left(t-t_{i}\right)$ and implies

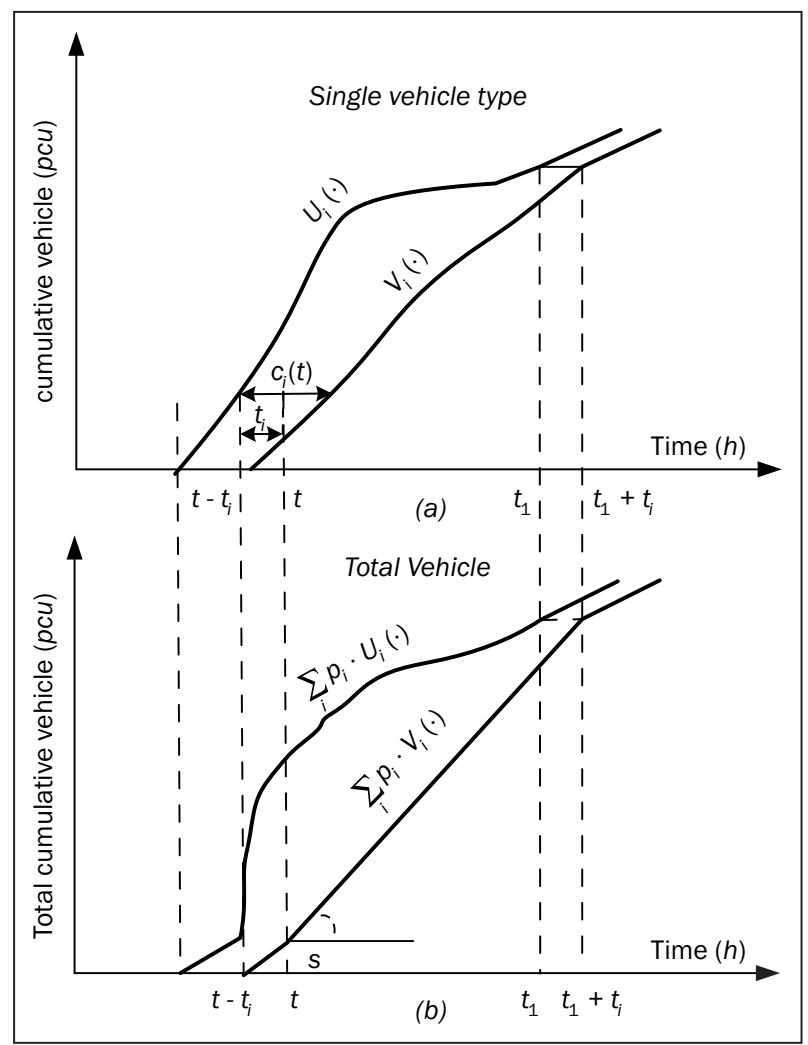

Figure 1 - Multiple vehicle type cumulative arrival and departure at a bottleneck constant running time ti required for a vehicle to arrive at the exit queue segment of a bottleneck link. The arrival flow rate of car (truck) travellers to the exit queue segment at the moment $t$ is $u_{i}\left(t-t_{i}\right)$ as well. The flow rate of car (truck) travellers exiting from the exit queue is $v_{i}(t)$. Thus, the link dynamic function can be written as follows.

$\frac{d q_{i}(t)}{d t}=u_{i}\left(t-t_{i}\right)-v_{i}(t), \forall i \in\{$ car, truck $\}, t \geq 0$,

where $q_{i}(t)$ is the number of car (truck) travellers waiting in the queue at moment $t$ on a bottleneck link.

Further, according to the integral of (1), one can see

$$
\begin{aligned}
& \int_{0}^{t} \frac{\mathrm{d} q_{i}(t)}{\mathrm{d} t} \mathrm{~d} t=\int_{0}^{t}\left(u_{i}\left(t-t_{i}\right)-v_{i}(t)\right) \mathrm{d} t \Rightarrow \\
& \Rightarrow q_{i}(t)=U_{i}\left(t-t_{i}\right)-V_{i}(t), \forall i \in\{c, t r\}, t \geq 0,
\end{aligned}
$$

where $U_{i}\left(t-t_{i}\right)$ expresses the cumulative arrival vehicle number of car (truck) travellers at a bottleneck until moment $t-t_{i}, V_{i}(t)$ expresses the cumulative departure vehicle number of car (truck) travellers at a bottleneck until moment $t, i \in\{c, t r\}$ expresses the abbreviated description that $c$ is for car and $t r$ is for truck as follows.

\section{Link Exit Flow Function}

If the sum of queue length of cars and trucks at a bottleneck link is

$$
\sum_{i \in\{c, t r\}} p_{i} \cdot q_{i}(t)>0
$$

or the sum of arrival flow rate of cars and trucks to the exit queue is

$$
\sum_{i \in\{c, t r\}} p_{i} \cdot u_{i}\left(t-t_{i}\right) \geq s(\text { in } P c u / h),
$$

then the arrival flow must wait because the maximal departure flow rate exiting a bottleneck link exit queue is s. A Passenger Car Equivalent $p_{i}$ compares with a passenger car the impact that every mode of transport has on traffic variables (such as speed, density). Thus, the link outflow rate of cars (trucks) can be calculated as follows.

$$
\begin{aligned}
& v_{i}(t)=\frac{u_{i}\left(t-t_{i}\right)}{\sum_{i} p_{i} \cdot u_{i}\left(t-t_{i}\right)} \cdot s, \forall i \in\{c, t r\}, t \geq 0 . \\
& \text { If } \sum_{i \in[c, t r]} p_{i} \cdot u\left(t-t_{i}\right)<s,
\end{aligned}
$$

then cars and trucks will pass the exit queue without delay. Thus, the link exit function can be formulated as follows.

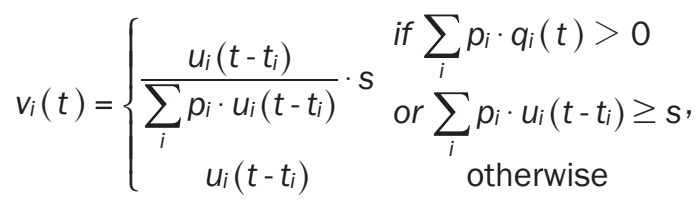

$\forall i \in\{c, t r\}, t \geq 0$ 


\section{Link Travel Time Function}

The total travel time on link a for cars (trucks) entering a bottleneck link at time $t$ can be given as

$c_{i}(t)=t_{i}+\frac{\sum_{m \in\{c, t r\}} p_{m} \cdot q_{m}\left(t+t_{i}\right)}{s}, \forall i \in\{c, t r\}, t \geq 0$.

The interested readers can further refer to [7], [8], [11] and [12].

\section{MULTIPLE VEHICLE TYPE DYNAMIC MARGINAL COST FUNCTION}

Following a similar analysis method presented by Kuwahara M. et al. and Li et al. in [3] and [11], the dynamic marginal cost of cars (trucks) at moment $t$ is given by taking the derivative of the total travel cost

$$
\alpha \cdot \sum_{i} \int_{0}^{T} u_{i}(t) \cdot c_{i}(t) \mathrm{d} t
$$

where $\alpha$ is the time-cost parameter, $T$ is the study horizon and derivative is done with respect to car (truck) demand $u_{i}(t) d t$ at moment $t$. Two cases of traffic conditions at a bottleneck link can be expressed as congested and uncongested conditions, respectively. The dynamic marginal travel cost of cars (trucks) under the two cases of traffic conditions can be expressed as below.

Case 1: when a car (truck) traveller entering a bottleneck link and caused a congested queue at the exit segment, $c_{c}(t)>t_{c}\left(c_{t r}(t)>t_{t r}\right)$ at moment $t$. Values $t_{c}$ and $t_{t r}$ express the free flow travel time of a car and truck on a bottleneck link, respectively. $t_{1}$ is total queue vanishing time. In other words, a car (truck) traveller entering a bottleneck link will experience a congested queue at interval $\left[t, t_{1}\right]$.

The dynamic marginal travel cost of a car (truck) can be equal to the derivative of total travel cost with respect to car (truck) demand in considering cars and trucks queuing property. Firstly, the derivation method on the dynamic marginal travel cost function of cars is given by following the similar method in [3, see (9a)].

$$
\begin{gathered}
M C_{c}(t)=\frac{d\left(\alpha \cdot \sum_{i} \int_{0}^{T} u_{i}(x) \cdot c_{i}(x) d x\right)}{d u_{c}(t) d t}= \\
=\frac{d\left(\alpha \cdot \int_{0}^{T} u_{c}(x) \cdot c_{c}(x) d x\right)}{d u_{c}(t) d t}+ \\
+\frac{d\left(\alpha \cdot \int_{0}^{T} u_{t r}(x) \cdot c_{t r}(x) d x\right)}{d u_{c}(t) d t}= \\
=\alpha \cdot \int_{0}^{T} c_{c}(x) \cdot \frac{d u_{c}(x)}{d d u_{c}(t)} \cdot \frac{1}{d t} \cdot d x+ \\
+\alpha \cdot \int_{t}^{t_{1}} \frac{d c_{c}(x)}{d u_{c}(t)} \cdot \frac{1}{d t} \cdot u_{c}(x) d x+
\end{gathered}
$$

$$
\begin{aligned}
& +\alpha \cdot \int_{0}^{T} c_{t r}(x) \frac{\mathrm{d} u_{t r}(x)}{\mathrm{d} u_{c}(t)} \cdot \frac{1}{\mathrm{~d} t} \cdot \mathrm{d} x+ \\
& +\alpha \cdot \int_{t}^{t_{1}} \frac{\mathrm{d} c_{t r}(x)}{\mathrm{d} u_{c}(t)} \cdot \frac{1}{\mathrm{~d} t} \cdot u_{t}(x) \mathrm{d} x
\end{aligned}
$$

Because

$$
\frac{\mathrm{d} u_{c}(x)}{\mathrm{d} u_{c}(t)}= \begin{cases}1, & x=t \\ 0, & x \neq t\end{cases}
$$

in the first term of the above equation, the first term becomes $\alpha \cdot c_{c}(t)$. Then

$$
\frac{\mathrm{d} u_{t r}(x)}{\mathrm{d} u_{c}(t)}=0
$$

the third term of the above equation equals zero. Meanwhile, the derivation

$$
\frac{\mathrm{d} c_{c}(x)}{\mathrm{d} u_{c}(t)}\left(\frac{\mathrm{d} c_{t r}(x)}{\mathrm{d} u_{c}(t)}\right)
$$

in the second and fourth terms of the above equation does not equal zero only in interval $\left[t, t_{1}\right]$ consisting of $[0, T]$, so we can obtain:

$$
\begin{aligned}
& M C_{c}(t)=\alpha \cdot c_{c}(t)+\alpha \cdot \int_{t}^{t_{1}} \frac{\mathrm{d} c_{c}(x)}{\mathrm{d} u_{c}(t)} \cdot \frac{1}{\mathrm{~d} t} \cdot u_{c}(x) \mathrm{d} x+ \\
& \quad+\alpha \cdot \int_{t}^{t_{1}} \frac{\mathrm{d} c_{t r}(x)}{\mathrm{d} u_{c}(t)} \cdot \frac{1}{\mathrm{~d} t} \cdot u_{t r}(x) \mathrm{d} x .
\end{aligned}
$$

Substituting (2) into (5), we can obtain:

$$
\begin{aligned}
c_{c}(t) & =t_{c}+\frac{p_{c} \cdot\left(U_{c}(t)-V_{c}\left(t+t_{c}\right)\right)}{s}+ \\
+ & \frac{p_{t r} \cdot\left(U_{t r}\left(t+t_{c}-t_{t r}\right)-V_{t r}\left(t+t_{c}\right)\right)}{S} \\
c_{t r}(t) & =t_{t r}+\frac{p_{t r} \cdot\left(U_{t r}(t)-V_{t r}\left(t+t_{t r}\right)\right)}{s}+ \\
+ & \frac{p_{c} \cdot\left(U_{c}\left(t+t_{t r}-t_{c}\right)-V_{c}\left(t+t_{t r}\right)\right)}{S}
\end{aligned}
$$

Then, substituting (7a-b) into (6b), one can see:

$$
\begin{aligned}
& M C_{c}(t)=\alpha \cdot c_{c}(t)+\frac{p_{c} \cdot \alpha}{\mathrm{s}} \cdot \int_{t}^{t_{1}} \frac{\mathrm{d} U_{c}(x)}{\mathrm{d} u_{c}(t)} \cdot \frac{1}{\mathrm{~d} t} \cdot u_{c}(x) \mathrm{d} x+ \\
& +\frac{p_{c} \cdot \alpha}{s} \cdot \int_{t}^{t_{1}} \frac{d U_{c}\left(t+t_{t r}-t_{c}\right)}{d u_{c}(t)} \cdot \frac{1}{d t} \cdot u_{t r}(x) d x= \\
& =\alpha \cdot c_{c}(t)+\frac{p_{c} \cdot \alpha}{s} \cdot \int_{t}^{t_{1}} u_{c}(x) d x+\frac{p_{c} \cdot \alpha}{s} \cdot \int_{t+t_{c}-t_{r r}}^{t_{1}} u_{t r}(x) d x= \\
& =\alpha \cdot\left(t_{c}+\frac{p_{c} \cdot\left(U_{c}(t)-V_{c}\left(t+t_{c}\right)\right)}{s}+\right. \\
& \left.+\frac{p_{t r} \cdot\left(U_{t r}\left(t+t_{c}-t_{t r}\right)-V_{t r}\left(t+t_{c}\right)\right)}{S}\right)+ \\
& +\frac{p_{c} \cdot \alpha}{s} \cdot\left(U_{c}\left(t_{1}\right)-U_{c}(t)\right)+ \\
& +\frac{p_{c} \cdot \alpha}{s} \cdot\left(U_{t r}\left(t_{1}\right)-U_{t r}\left(t+t_{c}-t_{t r}\right)\right)= \\
& =\alpha \cdot t_{c}+\frac{p_{c} \cdot \alpha}{s} \cdot\left(U_{c}\left(t_{1}\right)-V_{c}\left(t+t_{c}\right)\right)+ \\
& +\frac{\left(p_{t r}-p_{c}\right) \cdot \alpha}{s} \cdot U_{t r}\left(t+t_{c}-t_{t r}\right)-
\end{aligned}
$$




$$
-\frac{p_{t r} \cdot \alpha}{\mathrm{s}} \cdot V_{t r}\left(t+t_{c}\right)+\frac{p_{\mathrm{c}} \cdot \alpha}{\mathrm{s}} \cdot U_{t r}\left(t_{1}\right)
$$

From Figure 1 (b), a car entering a bottleneck from time $t$ to time $t_{1}$ will experience the congested queue from time $t+t_{c}$ to time $t_{1}+t_{c}$ and the following equation can be given:

$$
\begin{aligned}
s \cdot\left(t_{1}+t_{c}-t-t_{c}\right)=s \cdot\left(t_{1}-t\right)= \\
=\int_{t+t_{c}}^{t_{1}+t_{c}}\left(p_{c} \cdot v_{c}(x)+p_{t r} \cdot v_{t r}(x)\right) d x= \\
=p_{c} \cdot v_{c}\left(t_{1}+t_{c}\right)+p_{t r} \cdot v_{t r}\left(t_{1}+t_{c}\right)- \\
\quad-\left(p_{c} \cdot v_{c}\left(t+t_{c}\right)+p_{t r} \cdot v_{t r}\left(t+t_{c}\right)\right)
\end{aligned}
$$

A car or a track entering a bottleneck at queue vanishing time $t_{1}$ will not experience queues. Therefore, one can obtain $V_{c}\left(t_{1}+t_{c}\right)=U_{c}\left(t_{1}\right), V_{t r}\left(t_{1}+t_{c}\right)=U_{t r}\left(t_{1}\right)$, then substitute them into (9). We can see:

$$
\frac{p_{c}}{s}\left(U_{c}\left(t_{1}\right)-V_{c}\left(t+t_{c}\right)\right)=t_{1}-t-\frac{p_{t}}{s}\left(U_{t r}\left(t_{1}\right)-V_{t r}\left(t+t_{c}\right)\right)
$$

Substituting (10) into (8), the dynamic marginal travel cost function of cars can be written as follows:

$$
\begin{gathered}
M C_{c}(t)=\alpha \cdot t_{c}+\alpha \cdot\left(t_{1}-t\right)-\frac{p_{t r} \cdot \alpha}{s}\left(U_{t r}\left(t_{1}\right)-V_{t r}\left(t+t_{c}\right)\right)+ \\
+\frac{\left(p_{t}-p_{c}\right) \cdot \alpha}{s} U_{t r}\left(t+t_{c}-t_{t r}\right)-\frac{p_{t r} \cdot \alpha}{s} \cdot V_{t r}\left(t+t_{c}\right)+ \\
+\frac{p_{c} \cdot \alpha}{s} \cdot U_{t r}\left(t_{1}\right)=\alpha \cdot\left(t_{1}+t_{c}-t\right)-\frac{p_{t r} \cdot \alpha}{s} \cdot U_{t}\left(t_{1}\right)+ \\
+\frac{p_{c} \cdot \alpha}{s} \cdot U_{t r}\left(t_{1}\right)+\frac{\left(p_{t}-p_{c}\right) \cdot \alpha}{s} \cdot U_{t r}\left(t+t_{c}-t_{t r}\right)= \\
=\alpha \cdot\left(t_{1}+t_{c}-t\right)+\frac{\left(p_{t r}-p_{c}\right) \cdot \alpha}{s} \cdot\left(U_{t r}\left(t+t_{c}-t_{t r}\right)-U_{t r}\left(t_{1}\right)\right)= \\
=\alpha \cdot\left(t_{1}+t_{c}-t\right)+\frac{\left(p_{c}-p_{t r}\right) \cdot \alpha}{s} \cdot\left(U_{t r}\left(t_{1}\right)-U_{t r}\left(t+t_{c}-t_{t r}\right)\right)
\end{gathered}
$$

Following a similar analysis method, the dynamic marginal travel cost function of trucks can be expressed as below:

$$
\begin{aligned}
M C_{t r}(t) & =\alpha\left[t_{1}+t_{t r}-t+\frac{p_{t r}-p_{c}}{s} \cdot\left(U_{c}\left(t_{1}\right)-U_{c}\left(t+t_{t r}-t_{c}\right)\right)\right], \\
\forall t & \geq 0
\end{aligned}
$$

According to (11), multiple vehicle-type dynamic marginal cost function that includes only one single vehicle-type is $\alpha\left(t_{1}+t_{c}-t\right)$ and was presented by $\mathrm{Ku}$ wahara M. in [3]. If there is only one vehicle type, (11) will be entirely similar with the function given in [3], so the single vehicle-type dynamic marginal cost model is a special case of the model presented here. Because the Pcu value of a car is less than that of a truck, according to (11), we can obtain $M C_{c}(t)<\alpha\left(t_{1}+t_{c}-t\right)$ and from (12), we can see $M C_{t r}(t)>\alpha\left(t_{1}+t_{t r}-t\right)$. Therefore, the dynamic marginal cost of a car traveller entering a bottleneck link which experiences congested queues at the exit segment at moment $t$ must be less than that of a truck at moment $t$. The real conditions reflect that the marginal change of the total system cost, triggered by a car traveller at moment $t$ en- tering the bottleneck link and experiencing congested queues at the exit segment, must be less than that of a truck. According to (11-12), the dynamic marginal cost functions of cars and trucks are directly related with queue vanishing time $t_{1}$. A car or a truck entering a bottleneck link at the start time of a queue will experience a very large dynamic marginal cost. Although its personal cost may not be large, since it will result in a queue and it will induce delay of almost everyone after entering a bottleneck link externality ([3]).

Case 2: if a car (truck) traveller entering a bottleneck link at moment $t$ does not cause the congested queues at the exit segment, that is, if $c_{i}(t)=t_{i}$, then the last two terms in (6) will become 0 . The dynamic marginal cost function of cars (trucks) can be expressed as:

$M C_{i}(t)=\alpha \cdot t_{i}, \forall i \in\{c, t r\}, t \geq 0$.

\section{OPTIMAL TOLL}

The dynamic optimal toll of cars (trucks) at moment $t$ are the difference between dynamic marginal cost and link cost of cars (trucks) at moment $t$ (Kuwahara and Li et al. [3], [11]).

$\operatorname{Toll}_{i}(t)=M C_{i}(t)-\alpha \cdot c_{i}(t), \forall i \in\{c, t r\}, t \geq 0$

According to (11-12) the dynamic optimal toll of cars and trucks can be expressed as follows:

\section{Case 1:}

$$
\begin{aligned}
& \text { Toll }_{c}(t)=\alpha \cdot\left(t_{1}+t_{c}-t\right)+ \\
& +\frac{\left(p_{c}-p_{t r}\right) \cdot \alpha}{s}\left(U_{t r}\left(t_{1}\right)-U_{t r}\left(t+t_{c}-t_{t r}\right)\right)- \\
& \quad-\alpha \cdot c_{c}(t), \forall t \geq 0 \\
& \text { Toll tr }(t)=\alpha\left[t_{1}+t_{t r}-t+\right. \\
& \left.+\frac{p_{t r}-p_{c}}{s}\left(U_{c}\left(t_{1}\right)-U_{c}\left(t+t_{t r}-t_{c}\right)\right)\right]- \\
& -\alpha \cdot c_{t r}(t), \forall t \geq 0
\end{aligned}
$$

Case 2:

$\operatorname{Toll}_{i}(t)=0, \forall i \in\{c, t r\}, t \geq 0$

\section{OPTIMAL CONDITIONS}

The System Optimal (SO) conditions are expressed to maximize the traveller surplus, so the multiple type traveller surplus for the study time period can be expressed as below:

$$
\begin{aligned}
S= & S 1-S 2=\sum_{i} \int_{0}^{T} \int_{0}^{u_{i}(t)} D_{i}^{-1}(x, t) \mathrm{d} x \mathrm{~d} t- \\
& -\sum_{i} \int_{0}^{T} \alpha \cdot u_{i}(t) \cdot c_{i}(t) \mathrm{d} t
\end{aligned}
$$

where $S 1$ is total traveller benefit, S2 is system total cost, $D_{i}^{-1}(x, t)$ is the inverse demand function which is a function of inflow rates of cars and trucks, because dynamic demand is $x_{i}(t)=u_{i}(t) \mathrm{d} t([3])$. 
The User Equilibrium (UE) is obtained when no car (truck) traveller has an incentive to change their travel choices. Because of that, the travel cost of a car (truck) travellers must be the same at all times [3]. Hence, the user equilibrium conditions can be stated as follows:

$U=\sum_{i} \int_{0}^{T} \int_{0}^{u_{i}(t)} D_{i}^{-1}(x, t) \mathrm{d} x \mathrm{~d} t-\sum_{i} \int_{0}^{T} \int_{0}^{u_{i}(t)} \alpha \cdot c_{i}(x) \mathrm{d} x \mathrm{~d} t$

\section{ALGORITHM}

In order to solve the dynamic traffic states and dynamic marginal travel cost under the above system optimization and user equilibrium conditions, a similar Newton steepest descent algorithm is presented. Hence, the derivation of the objective function of SO and UE conditions (18-19) with respect to link demand can be expressed as below, respectively.

The derivation of the objective function of SO conditions (18) with respect to link demand is calculated in the manner of [3].

$$
\begin{gathered}
\frac{\mathrm{d} S}{\mathrm{~d} u_{i}(t) \mathrm{d} t}=\frac{\mathrm{d} \sum_{m \in[c, t r]} \int_{0}^{T} \int_{0}^{u_{m}(t)} D_{m}^{-1}(x, t) \mathrm{d} x \mathrm{~d} t}{\mathrm{~d} u_{i}(t) \mathrm{d} t} \\
-\frac{\mathrm{d} \sum_{m \in[c, t r]} \int_{0}^{T} \alpha \cdot u_{m}(t) \cdot c_{m}(t) \mathrm{d} t}{\mathrm{~d} u_{i}(t) \mathrm{d} t}= \\
=\frac{\mathrm{d} \int_{0}^{T} \int_{0}^{u_{i}(t)} D_{i}^{-1}(x, t) \mathrm{d} x \mathrm{~d} t}{\mathrm{~d} u_{i}(t) \mathrm{d} t}-M C_{i}(t)= \\
=D_{i}^{-1}(x, t)-M C_{i}(t)
\end{gathered}
$$

The derivation of the objective function of UE conditions (19) with respect to link demand is:

$$
\frac{\mathrm{d} U}{\mathrm{~d} u_{i}(t) \mathrm{d} t}=D_{i}^{-1}(x, t)-\alpha \cdot c_{i}(t)
$$

The detailed procedures are explained stepwise below.

Step 1 - Set the initial distribution of car and truck inflow rates $u_{i}^{k}(t)$ and $k=0$.

Step 2 - According to the inflow rate of car and truck, the travel time and queue length can be calculated by (1-5). When the queue vanishing time $t_{1}$ is found, then the travel cost and dynamic marginal cost of car and truck under system optimization and user equilibrium conditions will be calculated.

Step 3 - Flow update (SO using (20) and UE using (21))

$$
\begin{aligned}
& u_{i}^{k}(t)=u_{i}^{k-1}(t)-\gamma \cdot \frac{\mathrm{d} S}{\mathrm{~d} u_{i}^{k-1}(t) \mathrm{d} t} \\
& u_{i}^{k}(t)=u_{i}^{k-1}(t)-\gamma \cdot \frac{\mathrm{d} U}{\mathrm{~d} u_{i}^{k-1}(t) \mathrm{d} t} \\
& \text { Set } \eta_{k}=\frac{1}{k+1} .
\end{aligned}
$$

Step 4 - Convergence computing until

$$
\sum_{i, t}\left|u_{i}^{k}(t)-u_{i}^{k-1}(t)\right|<\varepsilon
$$

and then stop. Otherwise, set $k=k+1$, and go to Step 2.

\section{SIMULATION EXAMPLE}

Let us consider a bottleneck with its capacity of $2,500 \mathrm{Pcu} / \mathrm{h}$. The passenger car equivalent parameters of cars and trucks are 1 and 2, respectively. The time interval length is 0.2 (unit time). The free flow times of a car and truck are 0.6 and 1 (unit time). The inverse demand function is given as follows:

$$
\begin{gathered}
D_{i}^{-1}\left(u_{i}(t), t\right)=\frac{a_{0}}{M_{i}^{-1}(t)}\left(M_{i}^{-1}(t)-c_{i}(t)\right), \\
\forall i \in\{c, t r\}, t \geq 0,
\end{gathered}
$$

where $M_{i}^{-1}(t)$ means the costs with respect to the maximum potential demand rate of cars (trucks) at moment $t$ and $a_{0}$ is the parameter of cars (trucks).

Figure 2 shows the inflow rates for car and truck travellers under user equilibrium and system optimal conditions. We can find that the inflow rates of car

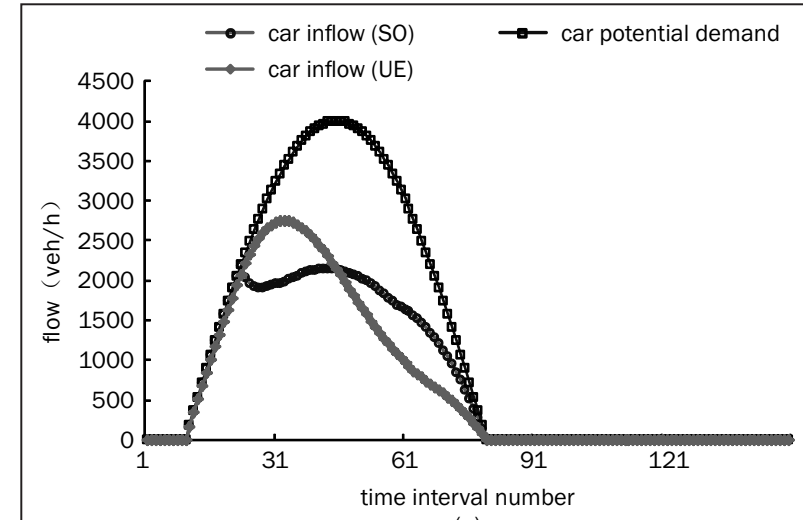

(a)

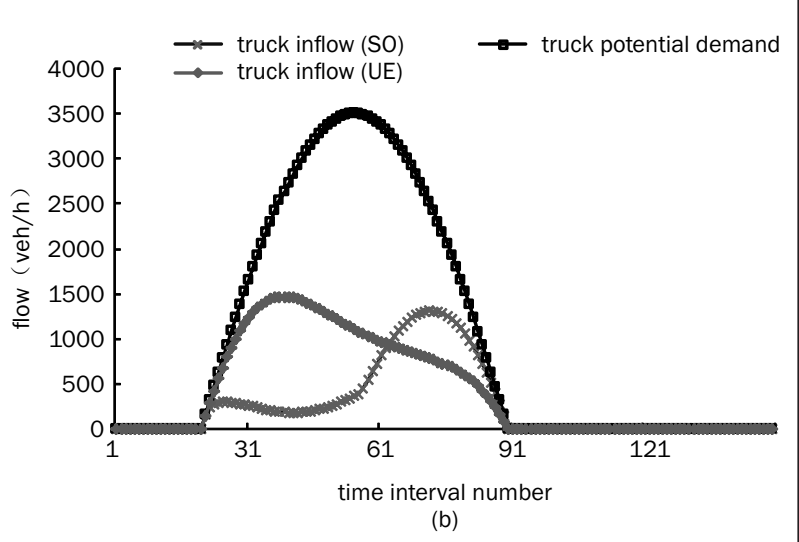

Figure 2 - Link inflow 


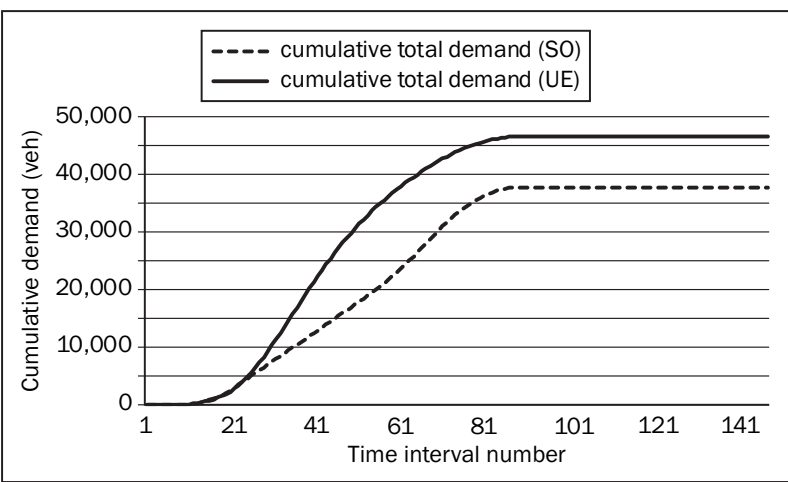

Figure 3 - Cumulative total demand at a bottleneck

and truck travellers under user equilibrium and system optimal conditions is less than the potential demand rate, and the inflow rates of car and truck travellers under user equilibrium conditions are not always greater than those under system optimal conditions. If two vehicle types are transformed into the standard car type, the cumulative total demand under system optimal conditions will be less than that under user equilibrium conditions as shown in Figure 3. It is similar with the analysis based on a single vehicle type, but it is shown that the analysis results based on single vehicle type cannot be simply extended to multiple vehicle types.

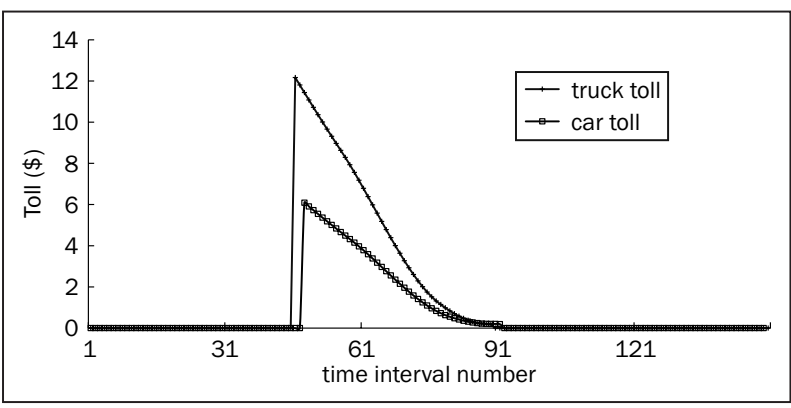

Figure 4 - Toll

Figure 4 gives the dynamic tolls of cars and trucks under system optimal condition. From this Figure, we can find that the toll of trucks is obviously larger than that of cars due to larger road space occupied by trucks. Further, although the inflow rate of cars can be greater than those of trucks, it does not influence that the toll of car is greater than the toll of trucks. As one can see, the toll of cars is lower.

According to the queue given in Figure 5, one can find that the queues of cars and trucks under SO conditions are smaller than those under UE conditions. Firstly, the cumulative total demand under SO conditions entering the network due to the congestion pricing will be less than that under UE conditions, according to Figure 3. Secondly, the inflow rates of cars and trucks with similar trend are relatively high in the beginning stage, and then gradually decrease under UE conditions (Figure 1). The conditions result in the simi-

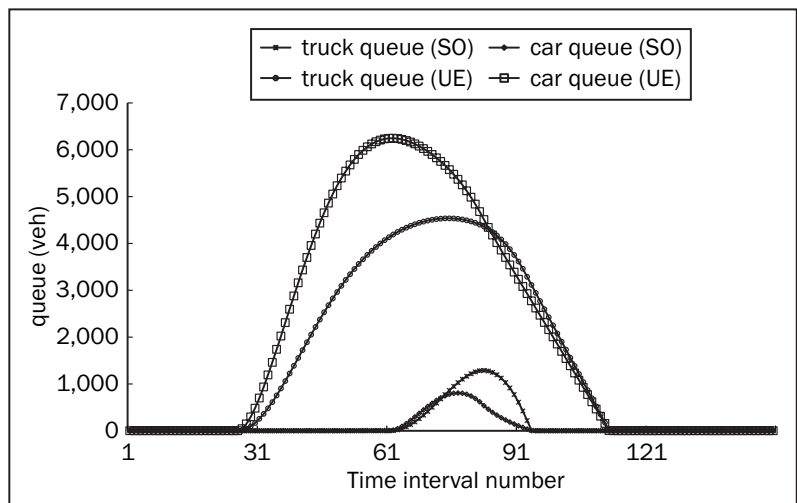

Figure 5 - Link queue

lar high peak of cars and trucks and further the larger queue at a bottleneck is induced. The change trend of the inflow rates of cars and trucks is opposite under SO conditions (Figure 1) and then the inflow rates of cars are relatively high in the beginning stage and then gradually decrease, while the trend with trucks is vice versa. It is seen that the high peak time of cars and trucks is effectively staggered, thus the queues at a bottleneck link can be reduced. This also explains why congestion pricing can reduce traffic congestion on a bottleneck link.

\section{CONCLUSION}

Multiple vehicle type point queue model is used to calculate the analytical dynamic marginal cost function in considering the interactions of cars and trucks at a bottleneck link without considering departure time choices. The analytical dynamic toll functions of cars and trucks are given and the algorithm under system optimal and user equilibrium conditions is presented. The simulation example shows that the analysis method of single vehicle type cannot be simply extended to multiple vehicle types and we can find the reduction of the demand and the staggered high peak times of cars and trucks result in the reduction of traffic congestion. Further studies will investigate:

1. possible applications of multiple vehicle type dynamic congestion pricing model analysed further in the congested bottleneck;

2. bottleneck-based multiple vehicle type dynamic congestion pricing function extended to the network-based model;

3. multiple vehicle type point queue dynamic marginal cost function extended into multiple vehicle type physical queue model.

\section{ACKNOWLEDGEMENT}

Financial support provided by the National Natural Science Foundation of China (60804049) and the Special Fund for Basic Scientific Research of Cen- 
tral Colleges provided by Chang'an University (CHD2010JC022, CHD2012DK09) is acknowledged. We are grateful to three anonymous referees for constructive comments.

\section{李曙光}

陕西省长安大学电子与控制工程学院 邮编：

710064

基于瓶颈的多车型动态边际费用模型与算法研究

本文将基于瓶颈的单一车型的动态动态边际费用模型扩展 为多车型模型。并给出了一个动态瓶颈路段模型模拟小汽 车和卡车之间的相互作用。路段分为两个部分：一个是运 行段, 小汽车和卡车可以根据自身的自由流运行速度行 驶; 另一部分是出口排队段, 假设车辆都是没有长度的 点。使用车辆换算系数将卡车转化为标准小汽车处理。因 此, 小汽车和卡车的出口流量可以根据瓶颈容量计算得 到。并依据动态瓶颈路段模型推导出小汽车和卡车的动态 边际费用函数的解析表达式。进一步给出了一个简化启发 式算法求解在系统优化和用户平衡状况下的相应的瓶颈排 队与收费状况。最终, 通过仿真实验验证了算法和模型的 简单性和有效性。

多车型; 动态边际费用函数; 点排队; 交通瓶颈

\section{LITERATURE}

[1] Vickrey, W.S., Congestion theory and transport investment. American Economic Review, 1969, 59(2):pp. 251-261

[2] Yang, H., HaiJun, Huang. Analysis of time-varying pricing of a bottleneck with elastic demand using optimal control theory. Transportation Research Part B: Methodological, 1997, 31(6): pp. 425-440

[3] Kuwahara, M., A theory and implications on dynamic marginal cost. Transportation Research Part A: Policy and Practice, 2007, 41 (5):pp. 627-643
[4] Arnott, R., Palma, A.D., Lindsey, R., Route choice with heterogeneous drivers and group-specific congestion costs. Regional Science and Urban Economics, 1992, 22(1):pp. 71-102

[5] Verhoef, E.T., Rouwendal, J., Rietveld, P., Congestion caused by speed differences. Journal of Urban Economics, 1999, 45(4):pp. 533-556

[6] Holguín-Veras, J., Cetin, M., Optimal tolls for multi-class traffic: Analytical formulations and policy implications. Transportation Research Part A: Policy and Practice, 2009,43(4):pp. 445-467

[7] Shuguang Li, Yanming-Su., Multimode Stochastic Dynamic Simultaneous Route/Departure Time Equilibrium Problem with Queues. Journal of the Eastern Asia Society for Transportation Studies, 2005, 6(1):pp. 2092-2107

[8] Shuguang Li, Qinghua Zhou, Analysis of Multi-Mode Dynamic Deterministic Point Model Properties in Dynamic Traffic Assignment, Journal of Highway And Transportation Research And Development, 2007, 23(8):pp. 200-207 (in Chinese)

[9] Li, J., Fujiwara, O. and Kawakami, S., A reactive dynamic user equilibrium model in a network with queues. Transportation Research. B 2000. 34(8): pp. 605-624

[10] Huang, H. and L. W.H.K, Modeling and Solving the Dynamic User Equilibrium Route and Departure Time Choice Problem in Network with Queues. Transportation Research B, 2002. 36(3): pp. 253-273

[11] Shuguang Li, Qinghua Zhou, A theory of multiple vehicle-type dynamic marginal cost with departure time choice, Transport. Vilnius: Technika, 2010, 25(3):pp. 307-313

[12] Shuguang Li, Xu, Hongke, Physical-Queue DiscreteTime Dynamic Network Loading with Multiple Vehicle Types, Journal of Transportation Systems Engineering and Information Technology, 2009, 9(1):pp. 56-61 